\title{
Mucus strands from submucosal glands initiate mucociliary transport of large particles
}

\author{
Anthony J. Fischer, ${ }^{1}$ Maria I. Pino-Argumedo, ${ }^{2}$ Brieanna M. Hilkin, ${ }^{2}$ Cullen R. Shanrock, ${ }^{1}$ \\ Nicholas D. Gansemer, ${ }^{2}$ Anna L. Chaly, ${ }^{1}$ Keyan Zarei, ${ }^{2,3}$ Patrick D. Allen, ${ }^{1}$ Lynda S. Ostedgaard, ${ }^{2}$ \\ Eric A. Hoffman, ${ }^{3,4}$ David A. Stoltz, ${ }^{2,3,5}$ Michael J. Welsh, ${ }^{2,5,6}$ and Mahmoud H. Abou Alaiwa ${ }^{2,3}$ \\ 'Department of Pediatrics, ${ }^{2}$ Department of Internal Medicine and Pappajohn Biomedical Institute, ${ }^{3}$ Department of \\ Biomedical Engineering, ${ }^{4}$ Department of Radiology, and ${ }^{5}$ Department of Molecular Physiology and Biophysics, Roy J. \\ and Lucille A. Carver College of Medicine, University of lowa, lowa City, lowa, USA. ${ }^{6}$ Howard Hughes Medical Institute, \\ University of lowa, lowa City, lowa USA.
}

\begin{abstract}
Mucus produced by submucosal glands is a key component of respiratory mucociliary transport (MCT). When it emerges from submucosal gland ducts, mucus forms long strands on the airway surface. However, the function of those strands is uncertain. To test the hypothesis that mucus strands facilitate transport of large particles, we studied newborn pigs. In ex vivo experiments, interconnected mucus strands moved over the airway surface, attached to immobile spheres, and initiated their movement by pulling them. Stimulating submucosal gland secretion with methacholine increased the percentage of spheres that moved and shortened the delay until mucus strands began moving spheres. To disrupt mucus strands, we applied reducing agents tris-(2-carboxyethyl)phosphine and dithiothreitol. They decreased the fraction of moving spheres and delayed initiation of movement for spheres that did move. We obtained similar in vivo results with CT-based tracking of microdisks in spontaneously breathing pigs. Methacholine increased the percentage of microdisks moving and reduced the delay until they were propelled up airways. Aerosolized tris-(2-carboxyethyl)phosphine prevented those effects. Once particles started moving, reducing agents did not alter their speed either ex vivo or in vivo. These findings indicate that submucosal glands produce mucus in the form of strands and that the strands initiate movement of large particles, facilitating their removal from airways.
\end{abstract}

Conflict of interest: The University of lowa Research Foundation has licensed intellectual property related to gene modified pigs to Exemplar Cenetics. Royalties from that license are shared with MJW and DAS. Neither MJW nor DAS have other financial ties to Exemplar Cenetics. EAH is a founder and shareholder of VIDA Diagnostics, a company commercializing lung image analysis software developed, in part, at the University of lowa.

License: Copyright 2019, American Society for Clinical Investigation.

Submitted: September 12, 2018 Accepted: November 29, 2018 Published: January 10, 2019

Reference information: JCI Insight. 2019;4(1):e124863. https://doi.org/10.1172/jci. insight.124863.

\section{Introduction}

Mucociliary transport (MCT) removes inhaled and aspirated particles and pathogens from lungs (1-3). This important respiratory host defense mechanism depends on mucus, which binds particulate material and carries it up the airways, propelled by ciliated surface epithelial cells $(1,3)$. Mucus contains a variety of antimicrobial factors and other proteins, but mucins are the major structural components (4). In human airways, the predominant secreted gel-forming mucins are MUC5AC and MUC5B (3-7). These are heavily glycosylated proteins linked end-to-end by disulfide bonds to form very long structures $(4,7,8)$.

In humans, pigs, and other large mammals, mucins are produced by proximal and distal airways. The proximal airways are cartilaginous, contain submucosal glands, and, in humans, extend through the first 10 generations to airways with a diameter of $1-2 \mathrm{~mm}$ (6). There are two sources of mucus in cartilaginous airways. The surface epithelium contains goblet cells and club cells that produce mucus, including MUC5AC and MUC5B $(3,9-11)$. Submucosal glands are also a major source of mucus (12-15). Within the gland's acinus, mucous cells produce MUC5B and very little or no $\operatorname{MUC5AC}(10,11,16)$. Gland secretion is regulated primarily by receptors on the cell's basolateral surface in response to neurohumoral stimuli, including cholinergic agonists $(6,17-19)$.

Ex vivo and in vivo studies of porcine airways have revealed that mucus emerges from submucosal gland ducts onto the airway surface in the form of strands $(16,20-22)$. Submucosal glands of humans also form strands $(23,24)$. The core of mucus strands is composed of MUC5B filaments, and MUC5AC often coats the surface of strands $(16,21,22)$. However, whether the strands per se facilitate MCT is uncertain.

Here, we hypothesized that mucus strands produced by submucosal glands facilitate MCT. To examine 
the normal function of mucus strands, we disrupted them using tris(2-carboxyethyl)phosphine (TCEP) or dithiothreitol (DTT). These reducing agents break the intermolecular disulfide bonds that join MUC5B from submucosal glands as well as MUC5AC and MUC5B from surface epithelia $(25,26)$. We studied pigs because their lungs and submucosal glands are similar to those of humans $(6,12,27)$. We examined the interaction of mucus strands with particles ex vivo and then, with that knowledge, assessed particle transport in vivo.

\section{Results}

Mucus strands emerge from submucosal gland ducts onto the tracheal surface. To investigate the macroscopic structure of submucosal gland mucus, we excised and submerged newborn pig trachea and examined the organization and behavior of individual mucus strands. We examined the airway under basal conditions and after stimulating submucosal gland secretion by adding the cholinergic agonist methacholine $(6,16,20)$. We labeled mucus strands with 40-nm fluorospheres. Consistent with earlier studies, individual mucus strands arose from submucosal gland ducts. Mucus strands progressively grew longer as they emerged from ducts, typically broke near the mouth of the duct, and were swept away (Figure 1A and Supplemental Video 1; supplemental material available online with this article; https://doi.org/10.1172/jci.insight.124863DS1) $(16,20,21)$. However, mucus strands occasionally stretched and broke and the proximal portion snapped back (Figure 1B and Supplemental Video 2). While connected to the duct, mucus strands sometimes twisted and rolled before they broke free (Supplemental Video 1).

While strands were still emerging from submucosal gland ducts, they sometimes associated with each other or with strands that were moving across the surface to form networks of mucus strands (Figure 1C and Supplemental Video 3). This occurred under both basal and methacholine-stimulated conditions. Once strands broke, releasing them from ducts, complex networks of mucus strands swept across the airway surface (Figure 1D and Supplemental Videos 4 and 5). Networks often included some strands that were oriented perpendicular to the direction of ciliary flow, crossing the epithelium like a squeegee.

Reducing agents disrupt submucosal gland mucus strands. Reducing agents, such as TCEP and DTT, break intermolecular disulfide bonds that hold mucins such as MUC5AC and MUC5B together $(25,26)$. Reduction of disulfide bonds by DTT is optimal at a $\mathrm{pH}$ of above 7 and may be reversible, whereas reduction by TCEP is irreversible and reactive under more acidic conditions due to its lower $\mathrm{pKa}(26)$.

To determine if reducing agents disrupt mucus strands, we examined methacholine-stimulated mucus secretion in the presence and absence of reducing agents. In the absence of reducing agent, submucosal glands produced mucus strands (Figure 2A). However, in the presence of DTT, mucus was released piecemeal as smaller fragments that were transported by ciliary beating and the resulting fluid flow (Figure $2 \mathrm{~A}$ ). We obtained similar results with $1 \mathrm{mM}$ TCEP.

We quantitated the effect of DTT and TCEP on mucus strands by counting the number of intact mucus strands that flowed over the tracheal surface during a 15-minute period. We counted intact strands that passed two points separated by $140 \mu \mathrm{m}$. Under control conditions, approximately 86 strands per 15 -minute period flowed over the surface (Figure $2 \mathrm{~B})$. Both TCEP $(1 \mathrm{mM})$ and DTT $(1 \mathrm{mM})$ decreased the number of strands. In contrast, ciliary beat frequency was unaffected by reducing agents (Figure $2 \mathrm{C}$ ). These data suggest that DTT and TCEP disrupt mucus strands without significantly affecting ciliary beating.

Mucus strands initiate particle transport. To test the contribution of mucus strands to MCT, we applied 500$\mu \mathrm{m}$ metallic spheres to the ex vivo tracheal surface. We used these spheres because they are easily visualized, their size approximates that of some aspirated particles, they have been used previously (28), and we suspected that mucus strands might be required to move them. We used time-lapse photography to examine trajectories of individual spheres in the presence or absence of reducing agents (Figure 3A and Supplemental Video 6). In the absence of reducing agents, many of the spheres dropped onto the airway surface were swept to the edges of the tissue. However, in the presence of $1 \mathrm{mM}$ TCEP or DTT (see Supplemental Figure 1), a greater number remained in their initial positions for the duration of the experiment. We quantified the percentage of particles that were cleared from the tracking field to the edge of the tissue. Compared with basal conditions, stimulating submucosal gland secretion with methacholine increased the percentage of spheres that left the field (Figure 3B). TCEP decreased the clearance of spheres under both basal conditions and after methacholine stimulation.

To understand why these spheres did not clear the tracking field, we assessed the movement of each particle individually. When we placed spheres on the airway surface, they often remained in place or sometimes rolled backward, driven by ciliary beating (Supplemental Video 7). However, after a lag time, mucus strands collided with spheres and attached to their surface. The strands extended beyond 
A
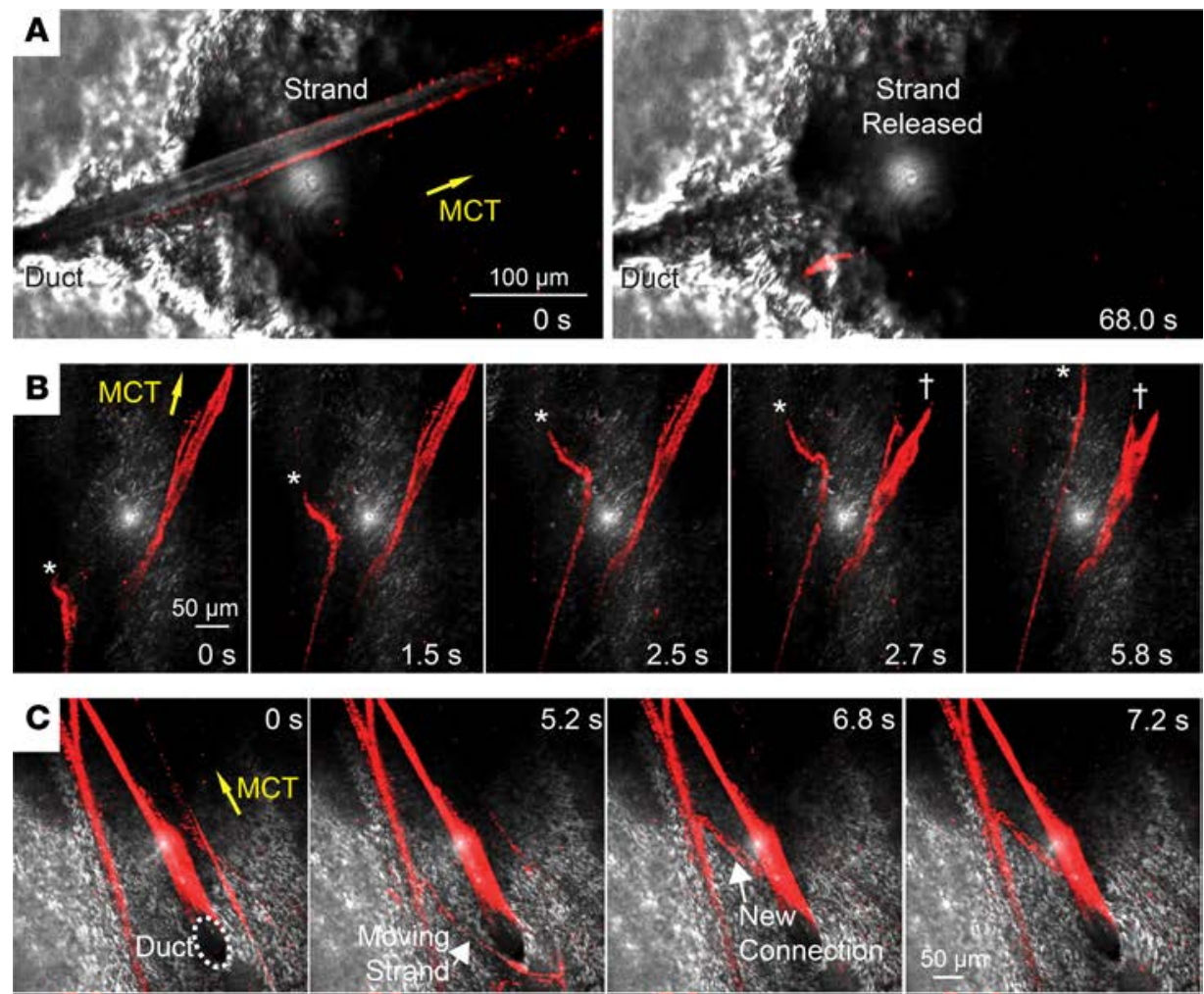

$0 \mathrm{~s}$
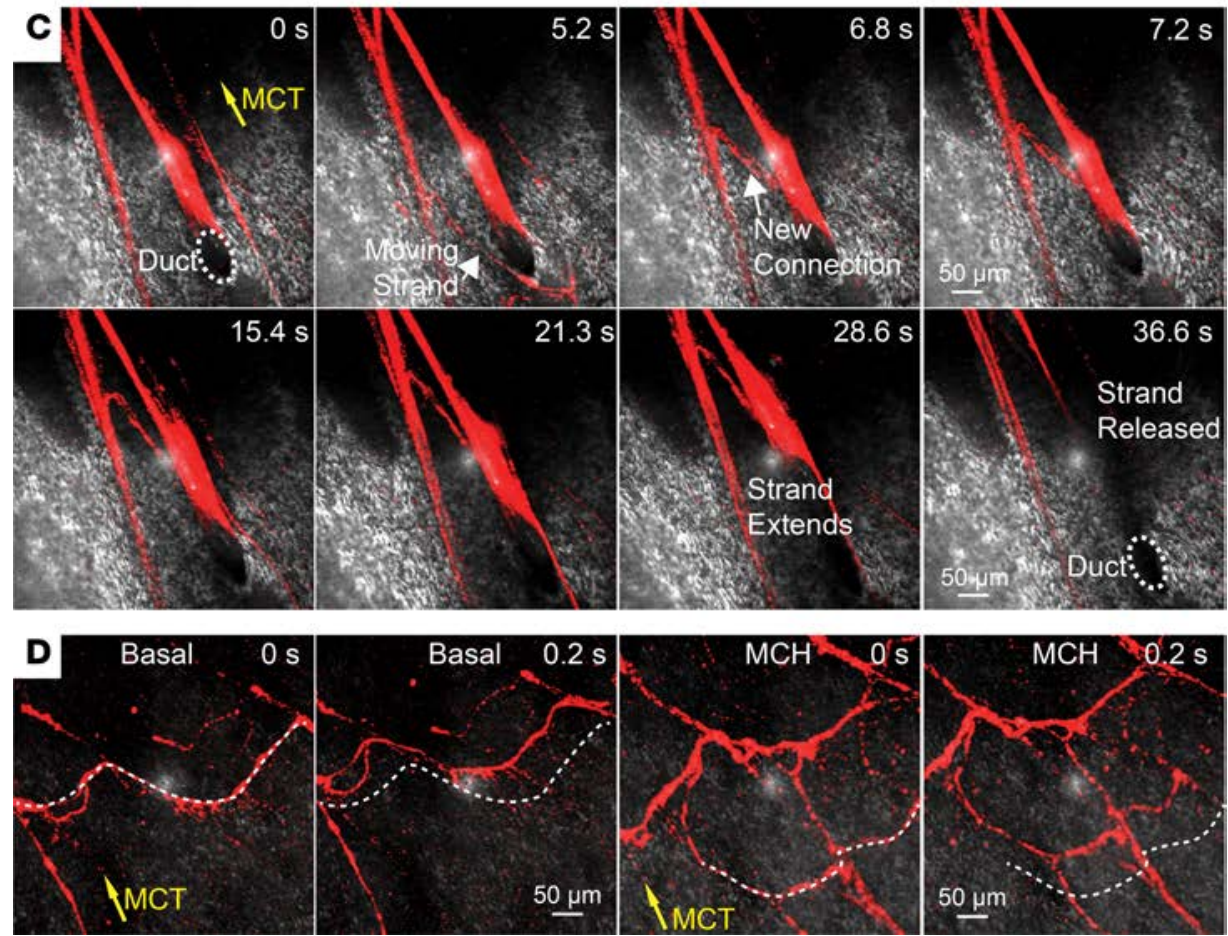

Figure 1. Mucus emerges from submucosal gland ducts as strands and forms networks. Confocal microscopic images of mucus strands labeled with 40-nm fluorospheres (red). Reflected light from airway surface is in gray; reflected light is the white dot in center of each image. Yellow arrows indicate the direction of MCT. Submucosal gland ducts are indicated with labels. In some experiments, submucosal gland mucus secretion was stimulated with methacholine $(\mathrm{MCH}, 100 \mu \mathrm{M})$. (A) A mucus strands emerges and is released from a submucosal gland duct. Scale bar: $100 \mu \mathrm{m}$. (B) Mucus strands break free from submucosal glands and move across the surface. The asterisk indicates the free end of a moving strand. The cross indicates a strand that was attached, breaks, and then abruptly snaps back. Scale bar: $50 \mu \mathrm{m}$. (C) Connection forming between adjacent moving strands and subsequent release of a strand from a duct. Scale bar: $50 \mu \mathrm{m}$. (D) Networks of mucus strands travel over the epithelial surface under basal and methacholine-treated conditions. The dotted line is reference to selected strands at time $=0$ seconds. Scale bar: $50 \mu \mathrm{m}$.

the sphere and eventually pulled them forward over the epithelial surface. Examples are provided in Figure 3C and Supplemental Videos 7 and 8. In contrast, in the presence of reducing agents, globules of mucus collided with spheres and attached to the surface (Figure 3D and Supplemental Videos 9 and 10). However, the accumulated mucus was often insufficient to stop the backward rolling or initiate sphere movement across the airway surface. We also assessed this behavior quantitatively. Once a strand attached to a sphere, it began moving after a short delay (Figure 3E). Moreover, the probability 

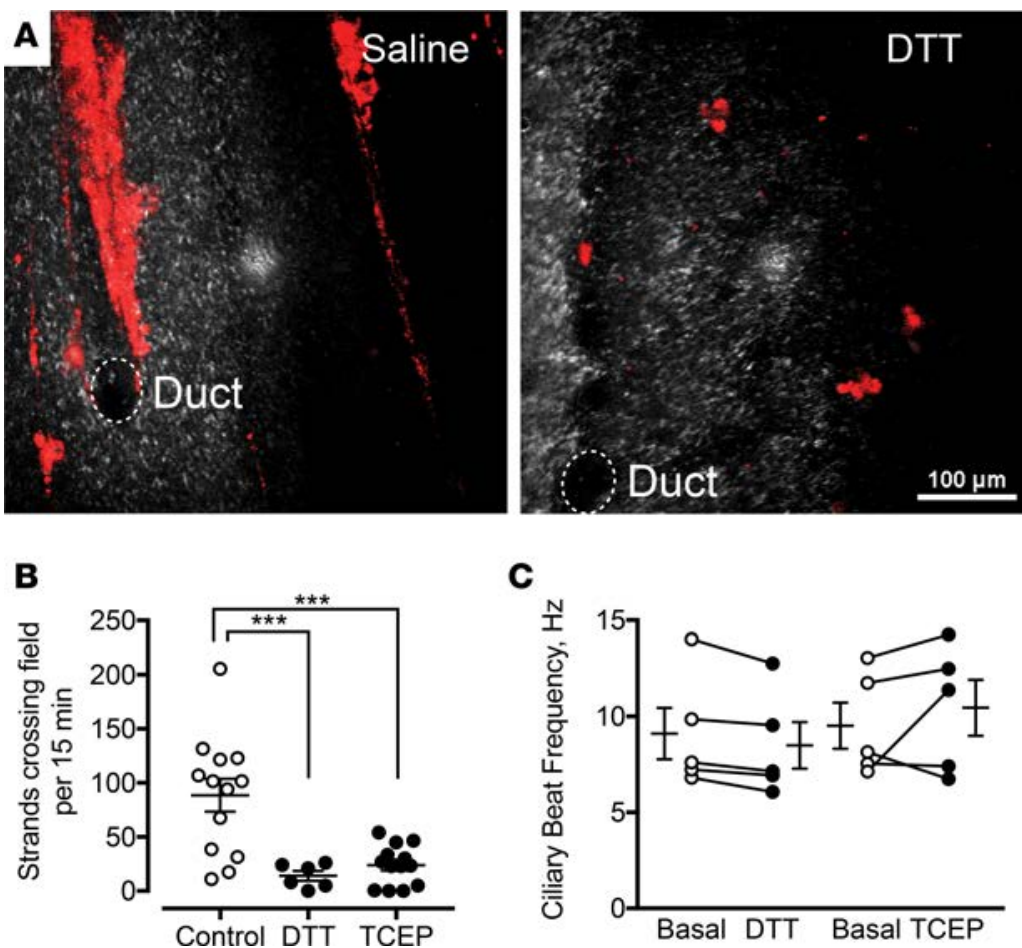

Figure 2. Reducing agents disrupt mucus strands. (A) Confocal microscopic images of mucus strands labeled with 40-nm fluorospheres (red). Reflected light is in gray. Submucosal gland ducts are indicated by dashed circles. Tissues were treated with methacholine and either vehicle or $1 \mathrm{mM}$ DTT. Scale bar: $100 \mu \mathrm{m}$. (B) Strands crossing the field of view in 15 minutes in the presence of $1 \mathrm{mM}$ DTT $(n=6)$ or TCEP $(n=13)$ compared with control $(n=13) .{ }^{* *} P<0.001$ by 1-way ANOVA. (C) Ciliary beat frequency under basal conditions before and after addition of $1 \mathrm{mM}$ DTT or $1 \mathrm{mM}$ TCEP $(n=5$ per group). Each set of data points and connecting lines is from a different pig. Lines and error bars represent mean \pm SEM.

of sphere movement when strands were attached was $>0.9$ (Figure $3 \mathrm{E}$ ). In contrast, in the presence of TCEP, the small globules of mucus had a low probability of moving spheres. The probability that small globules of mucus moved spheres was $<0.2$ (Figure $3 \mathrm{~F}$ ).

The pattern of movement for individual spheres showed substantial variability (29). Some spheres moved almost immediately after touching the surface of the epithelium. Many began moving after a delay. Some spheres were stationary for the duration of tracking; we refer to them as non-starters if they had not moved $>1 \mathrm{~mm}$ after at least 10 minutes of tracking (Figure 4A). We found that, under basal conditions, TCEP increased the percentage of non-starter spheres (Figure 4B). After stimulating submucosal gland secretion with methacholine, more of the spheres moved and the percentage of non-starters fell. However, in the presence of TCEP and methacholine, the percentage of non-starters increased to approximately $80 \%$ compared with approximately $20 \%$ with methacholine alone.

In addition to increasing the fraction of spheres that never moved (non-starters), for spheres that did move, TCEP prolonged the time until they moved $>1 \mathrm{~mm}$ from the original position. We measured the time elapsed between placing a sphere on the airway surface and its movement of $>1 \mathrm{~mm}$. Stimulating submucosal gland secretion with methacholine decreased the elapsed time (Figure 4, C and D). In airways treated with methacholine, TCEP more than doubled the elapsed time.

Once spheres began moving, TCEP did not significantly change the mean or maximum speed (Figure 4, E and F). We obtained similar results with DTT (Supplemental Figure 1). These results are consistent with the lack of effect of TCEP on ciliary beat frequency. These results suggest that mucus strands help initiate particle movement.

Aerosolized TCEP impairs MCT in vivo. Results from our ex vivo studies predicted that disrupting mucus strands would impair MCT in vivo. We therefore asked if aerosolized TCEP would prevent mucus strand formation in vivo. We administered methacholine i.v. and aerosolized vehicle (saline) or TCEP to pigs. Six minutes later, we removed the trachea, fixed it in $2 \%$ paraformaldehyde, and applied rhodamine-coupled 
A
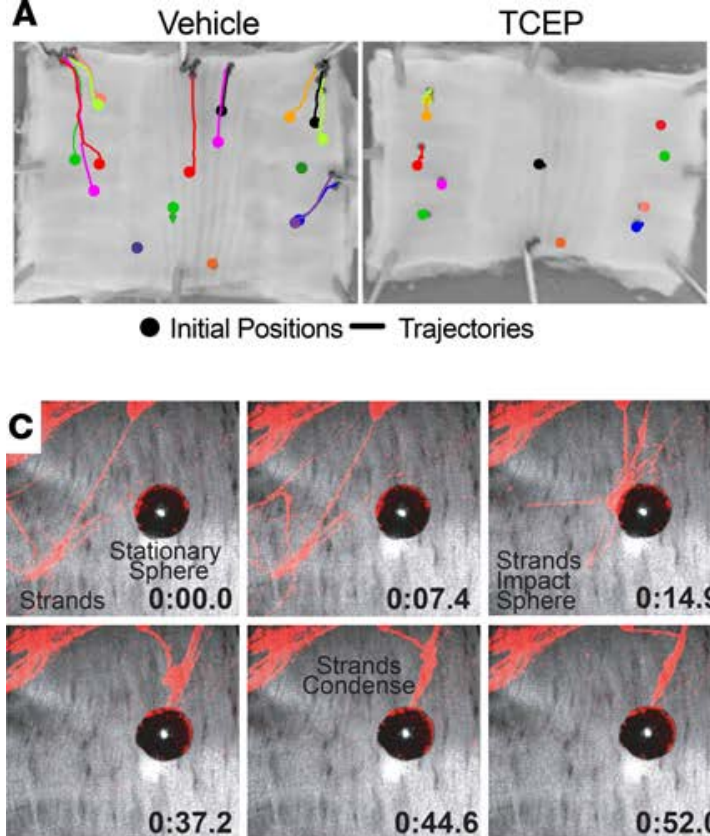

$0: 44.6$
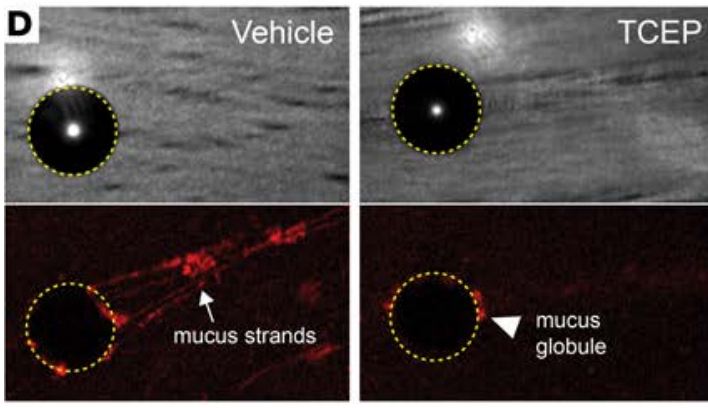

$\mathbf{F}$

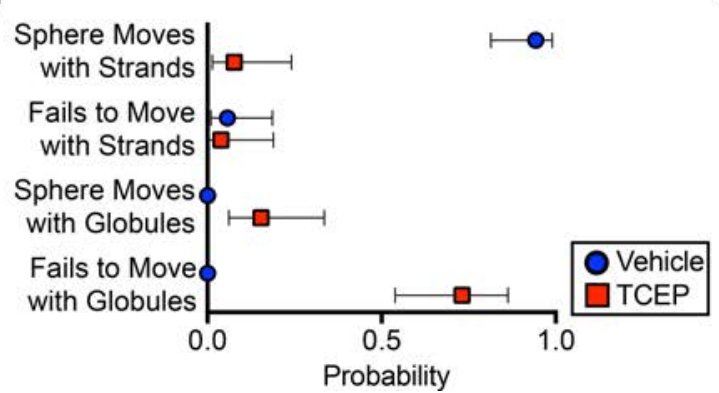

B
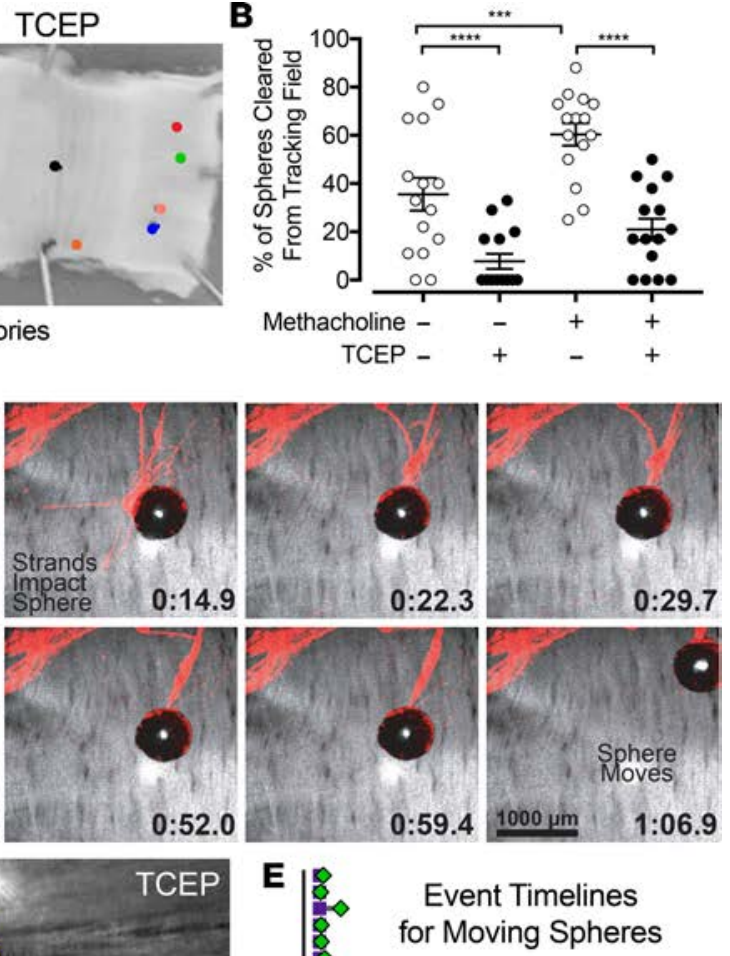

for Moving Spheres

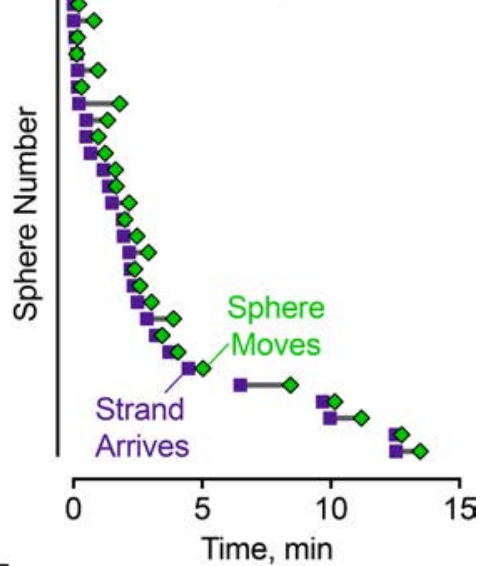

Figure 3. Reducing agents impair transport of spheres ex vivo. (A and B) Time-lapse photographic tracking of metallic spheres on segments of pig trachea in the presence or absence of reducing agents. (A) Images of ex vivo tracheal surface, with marks for initial positions (circles) and trajectories (lines) of spheres recorded over 15 minutes. The solution contained vehicle or $1 \mathrm{mM}$ TCEP. (B) Percentage of spheres cleared from the airway (i.e., moved to the edge of the tissue). $n=15$ per group; each data point represents a different pig. Lines and error bars represent mean $\pm S E M$. Some error bars are hidden by symbols. ${ }^{* *} P<0.001$, ${ }^{* * *} P<0.0001$ by 2 -way repeated-measures ANOVA with Holm-Sidak's post hoc multiple comparisons test. (C and $\mathbf{D})$ Confocal microscopic images from methacholine-stimulated airways of mucus strands labeled with 40-nm fluorospheres (red). Reflected light is gray. Metallic spheres appear black. (C) Time course of mucus strands initiating movement of a stationary sphere. Dorsal surface of airways is in middle. Scale bar: $1000 \mu \mathrm{m}$. (D) Mucus attachment to spheres in the presence of vehicle or $1 \mathrm{mM}$ TCEP. Top panels are reflected light images. Bottom panels show mucus labeled with fluorospheres (red). Dotted yellow circles mark outline of metallic spheres. The arrow marks mucus strands. The arrowhead marks mucus globules. Original magnification: $\times 4$. (E) Timelines from methacholine-stimulated airways showing arrival of mucus strands (purple squares) and movement of spheres (green diamonds) ( $n=33$ spheres from 10 different pigs). (F) Probability of spheres moving in the presence of methacholine $(100 \mu \mathrm{M})$ and the presence of vehicle ( $n=35$ spheres from 10 different pigs) or 1 mM TCEP ( $n=26$ spheres from 7 different pigs). Spheres were tracked for at least 15 minutes or until they moved. Symbols and error bars represent probability and $95 \% \mathrm{Cl} . P<0.0001$ by $\chi^{2}$ test. 

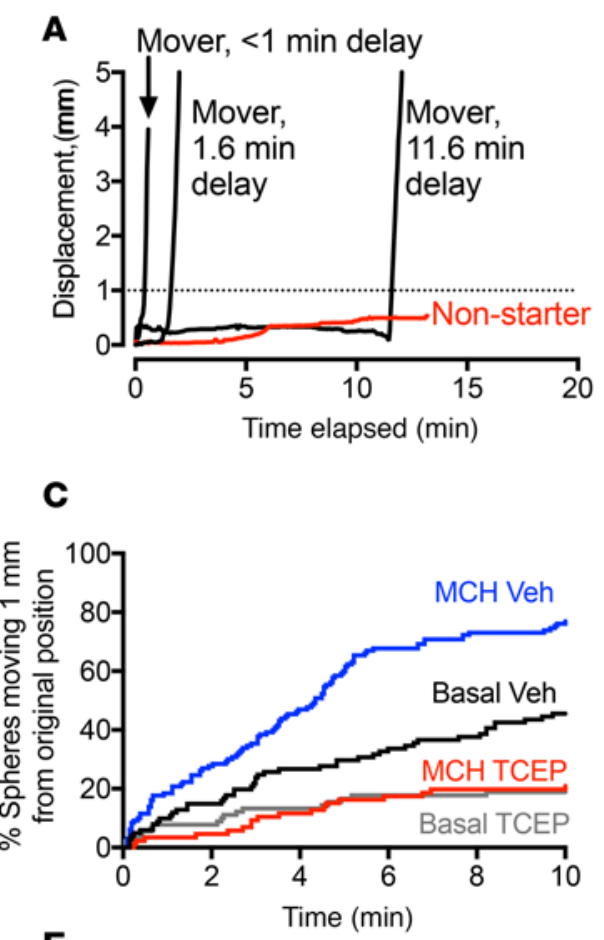

$\mathbf{E}$

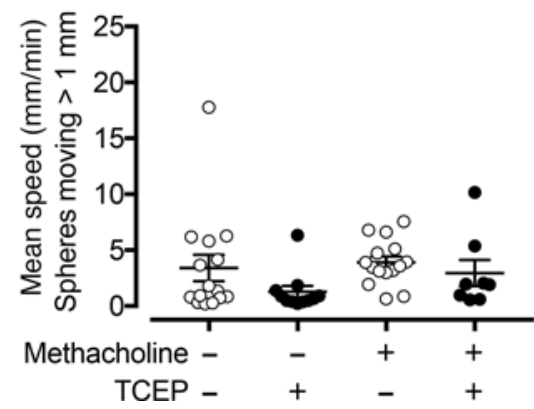

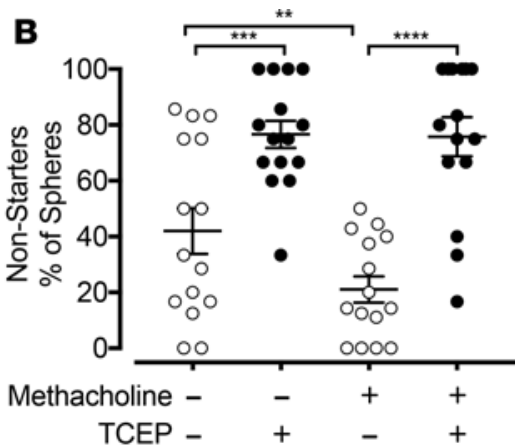

D
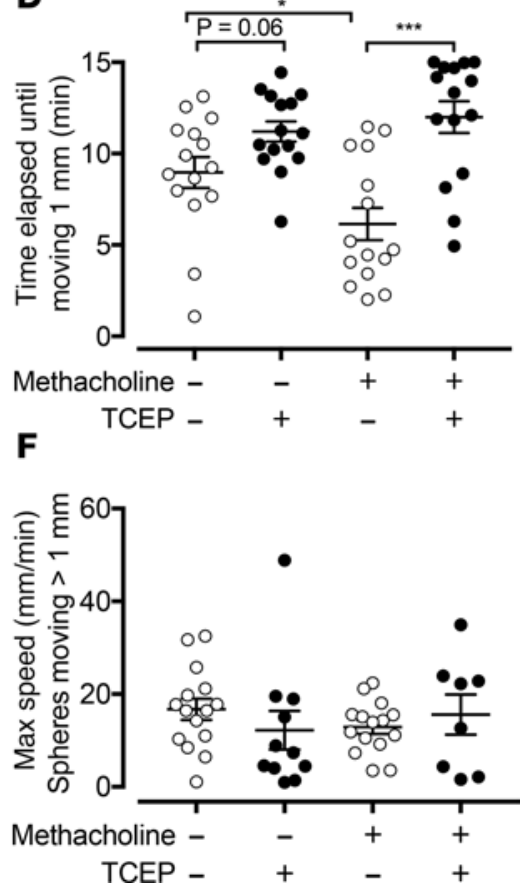

Figure 4. Reducing agents disrupt initiation of transport of spheres ex vivo. Time-lapse photographic tracking of metallic spheres on segments of pig trachea in the presence or absence of reducing agents. (A) Examples showing the displacement from original position as a function of time of 4 spheres. Some spheres moved immediately, and others had a variable delay before moving. Some spheres did not move (non-starters, red). A dotted line marks the 1-mm threshold for movement. (B) Percentage of non-starters in the presence and absence of methacholine (100 $\mu \mathrm{M})$ and the presence of vehicle or TCEP $(1 \mathrm{mM})$. Spheres were tracked for at least 10 minutes or until they moved. $n=15$ per group. Each data point is from a different pig. (C) Percentage of spheres moving $>1 \mathrm{~mm}$ from original position as a function of time. Data are pooled from 15 experiments, with 86-130 spheres tracked per condition. Conditions are basal/vehicle (black), basal/TCEP (gray), methacholine/vehicle (blue), and methacholine/TCEP (red). (D) Time elapsed before spheres moved $>1 \mathrm{~mm}$ from original position. $n=15$ per group. Each data point is the mean from a single pig. ( $\mathbf{E}$ and $\mathbf{F}$ ) Mean and maximum speed of moving spheres. $n=8-15$ (some conditions had no spheres moving $>1 \mathrm{~mm}$ ). Each data point is from a different pig. Lines and error bars represent mean $\pm \mathrm{SEM}$. Some error bars are hidden by symbols. ${ }^{*} P<0.05$, ${ }^{* *} P<0.01$, ${ }^{* * *} P<0.001$, ${ }^{* * *} P<0.0001$ by 2 -way repeated-measures ANOVA with Holm-Sidak's post hoc multiple comparisons test in (B and $\mathbf{D}$ ) or Kruskal-Wallis test for nonnormally distributed data in (E and $\mathbf{F}$ ).

wheat germ agglutinin (WGA) to label submucosal gland mucus. WGA predominantly labels MUC5B, and most MUC5B originates from submucosal glands in pigs and humans (16). Mucus strands were present on airways of vehicle-treated pigs, consistent with earlier reports (Figure 5A) (20). In contrast, TCEP-treated airways revealed areas that were devoid of mucus or had only small patches of mucus (Figure 5A). We also collected mucus, touched it to a slide, and stained it with the rhodamine-labeled WGA. We observed mucus strands from vehicle-treated airways (Figure 5B). However, in pigs that inhaled TCEP, the mucus had a watery quality, and the slide preparations showed small mucus blobs and fragments. 

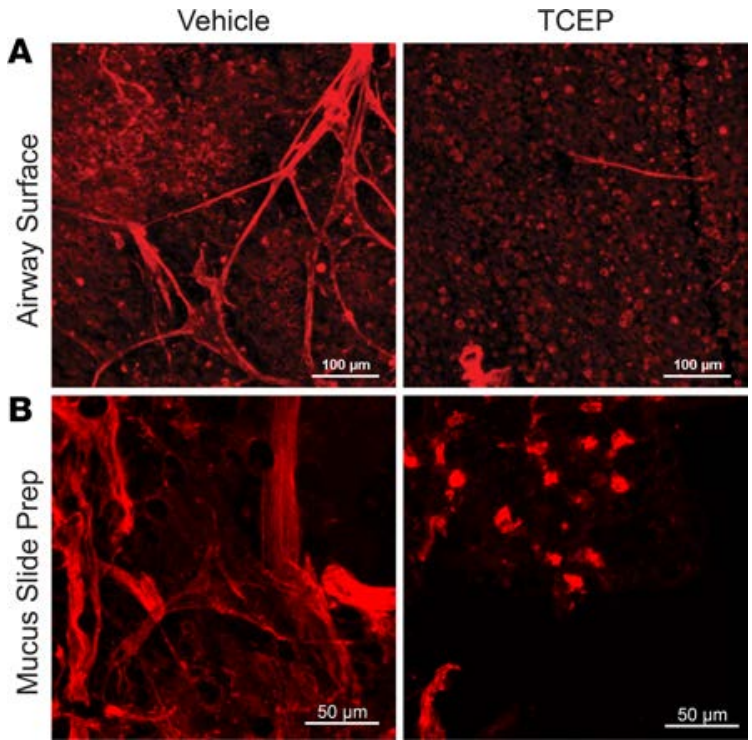

Figure 5. TCEP disrupts mucus strands in vivo. Fluorescence micrographs obtained after in vivo treatment with i.v. methacholine (MCH, $0.25 \mathrm{mg} / \mathrm{kg}$ ) and inhalation of vehicle ( $0.5 \mathrm{ml}$ saline) or TCEP (20 mM in $0.5 \mathrm{ml}$ saline). (A) Images of fixed segments of trachea. MUC5B-positive goblet cells are present in background. Scale bar: $100 \mu \mathrm{m}$. (B) Images of mucus collected in vivo and smeared on a slide. Mucus was stained with rhodamine-conjugated wheat germ agglutinin (red), which labels MUC5B. Scale bar: $50 \mu \mathrm{m}$.

We measured MCT in vivo before and after stimulating submucosal gland secretion with i.v. methacholine. To determine the role of mucus strands on MCT, we aerosolized TCEP or saline vehicle concurrently with methacholine. To measure MCT, we insufflated radiodense tantalum microdisks $(350 \mu \mathrm{m})$ into airways of sedated pigs, as previously described $(20,29)$. The pigs breathed spontaneously and were not intubated during the study. We obtained a high-resolution CT scan every 9 seconds for 6.3 minutes (total 44 scans). Figure 6A shows the trajectories of microdisks in 2 pigs, contrasting the effect of inhaled vehicle and TCEP. During the 6.3-minute scanning period, MCT cleared approximately $31 \%$ of the microdisks from the lungs under untreated conditions (Figure 6B). Methacholine more than doubled that percentage to approximately 74\%. However, aerosolized TCEP prevented methacholine from increasing microdisk clearance.

Tracking individual microdisks revealed considerable variability in transport under baseline and treated conditions (Supplemental Videos 11-13). Similar to our observations ex vivo, some microdisks moved immediately and others had variable delay (Figure 6C). To determine whether the failure to clear microdisks was related to failed initiation of transport, we classified microdisks as non-starters or movers, as we had done for ex vivo studies. We defined non-starters as microdisks that failed to move $>10 \mathrm{~mm}$.

Stimulating submucosal gland secretion with i.v. methacholine increased the fraction of microdisks that moved, and after methacholine, the percentage of non-starters was approximately $10 \%$ (Figure $6 \mathrm{D}$ ). However, after TCEP inhalation, methacholine failed to increase the fraction moving, and approximately $41 \%$ of microdisks were non-starters.

We measured the time required for individual microdisks to move, defined by displacement $>10 \mathrm{~mm}$ from the original position (Figure $6 \mathrm{E}$ ). Figure $6 \mathrm{E}$ shows the time it took for individual microdisks to move $>10 \mathrm{~mm}$, with data pooled by experimental treatment. Under basal conditions, individual microdisks initiated movement throughout the tracking period. When methacholine and inhaled vehicle or methacholine and inhaled TCEP were given, most microdisks initiated movement within the first minute. However, with methacholine and inhaled TCEP, fewer microdisks moved.

Using this method, we determined the elapsed time until microdisks moved $>10 \mathrm{~mm}$ from the original position for each experiment (Figure 6F). Under basal conditions, the time elapsed was approximately 3.2 minutes. This time was shortened to 1.1 minutes in the airways of pigs treated with methacholine and inhaled saline vehicle control. However, inhaled TCEP prolonged the time elapsed to approximately 3.2 minutes. Once microdisks began moving, TCEP had no effect on their velocity compared with saline vehicle (Figure 6, G and H). In the absence of submucosal gland stimulation, the effect of TCEP was minimal, consistent with TCEP acting on a product of submucosal glands (Supplemental Figure 2). 
A

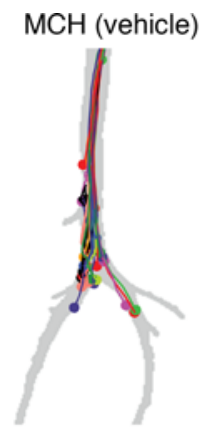

Initial Positions

C

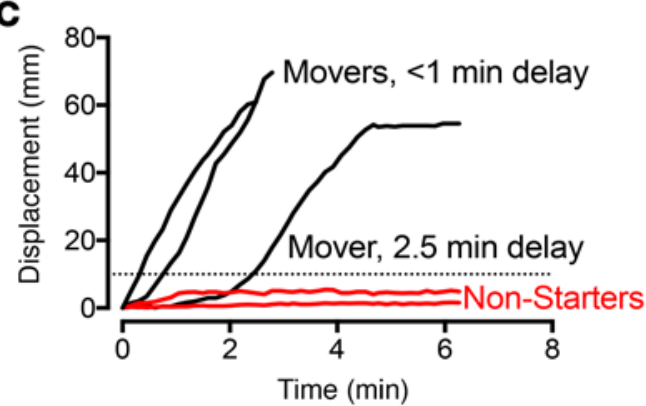

E

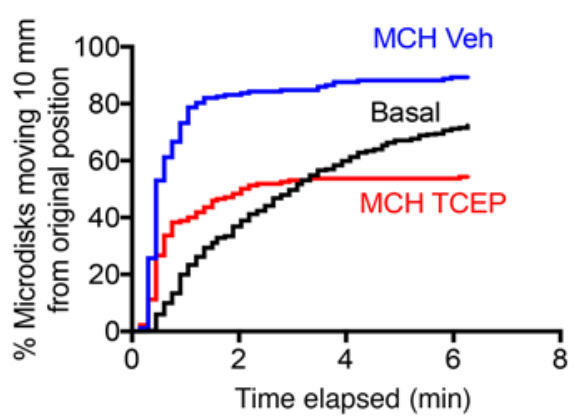

G

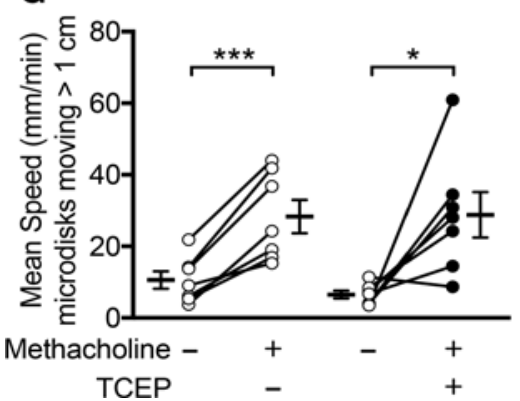

B

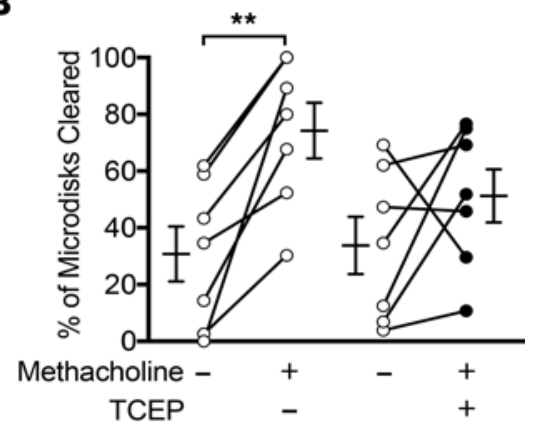

D

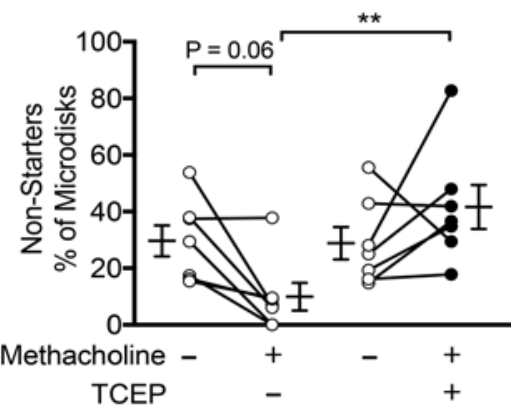

$\mathbf{F}$

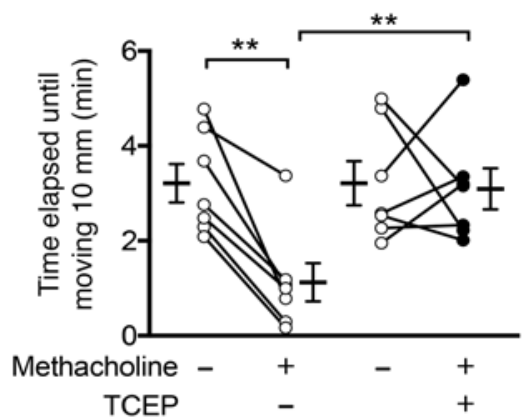

H

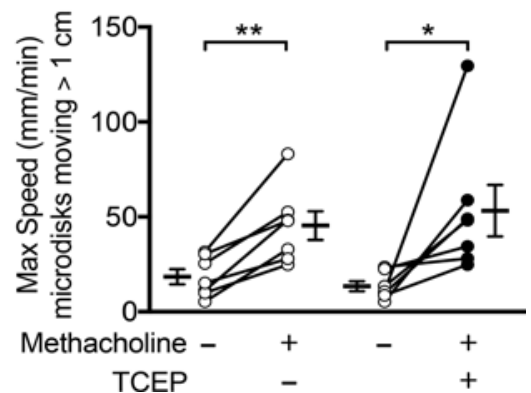

Figure 6. TCEP decreases methacholine-driven initiation of microdisk movement in vivo. In vivo tracking of tantalum microdisks using CT-based assay. (A) Examples showing initial positions and trajectories of microdisks in a pig that received i.v. methacholine and inhaled vehicle and a pig that received i.v. methacholine and inhaled TCEP. (B) Percentage of microdisks that were cleared from the lungs during the 6.3-minute tracking period. (C) Examples show displacement as a function of time of 5 microdisks. Some microdisks moved immediately, and others had a variable delay. Some microdisks never moved past the 10-mm threshold (dotted line) and are marked "non-starters" (red). (D) Percentage of microdisks that are non-starters. Data were obtained under basal conditions or after administration of i.v. methacholine and after aerosolized vehicle (left) or aerosolized TCEP (right). (E) Percentage of microdisks moving $>10 \mathrm{~mm}$ from original position as a function of time. Data are pooled from 7 experiments, with 178-354 microdisks tracked per condition. Conditions are basal (black), methacholine/vehicle (blue), and methacholine/TCEP (red). (F) Time elapsed before microdisks moved $>10 \mathrm{~mm}$ from original position. ( $\mathbf{G}$ and $\mathbf{H}$ ) Mean and maximum speed of moving microdisks. Each set of data points and connecting lines is from a different pig. Lines and error bars represent mean \pm SEM. $n=7$ per group. ${ }^{*} P<0.05$, ${ }^{* *} P<0.01$, ${ }^{* * *} P<0.001$ by 1-way ANOVA with Holm-Sidak's post hoc multiple comparisons test. 


\section{Discussion}

A major function of submucosal glands versus goblet cells is thought to be that submucosal glands can acutely release more mucus, especially in response to potentially harmful challenges and neurohumoral stimulation $(6,17,18,30)$. Our results suggest an additional function: submucosal glands produce mucus strands, which bind to particles and initiate their movement, thereby facilitating their clearance up the airways. Thus, mucus strands produced by submucosal glands may play an important defense function.

Our combined ex vivo and in vivo results identify events that occur when particles deposit onto the airway surface. The particles may make contact with cilia, as evidenced by the backward rotation of stationary spheres. But the beating of cilia alone is often not sufficient to move them forward. After mucus strands and networks are released from submucosal gland ducts, they travel across the airway surface and collide with and attach to the particles. They then began to pull particles, initiating transport up the airways.

There are several key factors in this sequence. Individual mucin molecules must interact to form long mucus strands and complex networks. Mucus strands must adhere to particles and they must be propelled by cilia. The importance of the mucus strands is indicated by the finding that when a cholinergic agonist increases production of mucus strands, the delay between deposit of a particle and its movement shortens and the percentage of particles that fail to move falls. The mucus strands and networks may move particles by increasing the effective area of contact with beating cilia and thereby increasing the force for movement. A critical role for strands was also evidenced when we reduced strand formation by disrupting disulfide bonds that connect mucin molecules; the percentage of particles that failed to move increased and the delay until particles began to move lengthened. This occurred even though mucus fragments could attach to particles and ciliary beating was intact.

Widdicombe and Wine (6) previously noted that the distribution of submucosal glands closely parallels the impact pattern for the largest inhaled particles. In addition, the number of submucosal gland duct openings and the volume of glands in mammalian airways correlate with airway size $(12,31)$. Those correlations together with our data suggest that submucosal glands and hence mucus strands may have evolved to protect against large particles, including aspirated and inhaled materials that deposit in cartilaginous airways. Widdicombe and Wine have suggested that small mammals such as rodents lack abundant submucosal glands in the trachea and pulmonary airways because large particles are filtered out in the nasopharynx and never reach the lungs (6). Thus, there may be less need for submucosal glands and mucus strands in small mammals.

Human and porcine airways secrete two gel-forming mucins that share substantial domain and sequence similarity, MUC5B and MUC5AC $(4,7)$. Whether they have distinct roles in MCT is unknown. Our data show that mucus strands are critical for removing particles, and previous work showed that MUC5B forms the core of the strands and MUC5AC associates with and sometimes coats the MUC5B core $(16,21,22)$. Thus, we wondered if MUC5B might provide strands with greater tensile strength and MUC5AC might provide strands with greater adhesive properties for binding particles. Together they might produce the most efficient MCT. It is interesting, however, that, in mice, which have only a few glands near the larynx, disrupting the Muc5b gene results in mucus plugs in the nasopharynx (32). That result suggested the MCT might be impaired, and studies of cultured epithelia were consistent with that possibility. Perhaps even more uncertain is the contribution of these two mucins to MCT in distal, noncartilaginous airways, which lack submucosal glands.

Here, we focused on large particles. That leaves unanswered the question of whether mucus strands facilitate clearance of small particles. Previous studies show that loss of baseline secretion of airway mucus can impair clearance of smaller particles in mice (32-34). Cilia can drive transport of small (40 nm to 4.5 $\mu \mathrm{m})$ particles in the presence of minimal mucus (35). These results suggest that mucus strands might not be required for transport of small particles, such as bacteria and viruses. However, the adhesiveness of mucus and the squeegee-like movement of mucus strands across the airway surface suggest that mucus strands might also contribute to MCT of small particles.

Earlier reports showed that reducing agents, including N-acetylcysteine, DTT, and TCEP, decrease mucus viscosity and elasticity (36-41). Although we are not aware of studies that investigated the effect of reducing agents on mucus strands, several studies reported effects of reducing agents on MCT. Some studies reported that reducing agents increased the speed of MCT $(37,38,42-46)$, whereas others reported that they slowed MCT $(28,39,41)$. There are many potential explanations for differences among studies. Species differences and the presence or absence of submucosal glands, airway anatomy, and epithelial cell types could influence results. In vivo studies could differ from studies of cultured epithelia, which lack submucosal glands and in which liquid and mucus cannot enter or leave the chamber. Humidification by a 
spontaneously breathing animal versus that provided to an intubated animal could affect results. Methodological differences could contribute; for example, methods could deposit particles in different parts of the lung, including the alveoli. The dose and/or type of reducing agent might be important $(43,47)$. The presence or absence of disease might also affect outcomes. Finally, it is interesting to consider that many studies of reducing agents have been performed with WT animals. Presumably WT animals have MCT properties that are optimal for that species; thus, either slowing or accelerating MCT might reduce efficiency.

This study has a number of advantages. First, the methods and analyses for tracking individual particles in vivo and ex vivo provided high spatial and temporal resolution $(20,29)$. That enabled us to determine whether particles were moving or not, measure time elapsed before particles began moving, and assess speeds of discrete particles. In contrast, techniques using small, diffusely distributed particles cannot measure these properties. For example, they cannot distinguish whether particles uniformly move at a slow speed or a small fraction of particles move quickly but a larger fraction of particles do not move at all. Second, we studied pigs, which have abundant submucosal glands that are similar to those in humans, and we tested the effect of stimulating submucosal gland secretion $(6,12)$. In addition, the animals breathed spontaneously through the nose and thus had normal airway anatomy, humidity, and airway surface composition. The pigs were newborns, which avoids potential airway remodeling, inflammation, and/or infection. Third, we assessed the role of mucus strands both ex vivo and in vivo. Thus, we were able to compare the data to identify interactions of individual particles with mucus strands. This approach also offered relevance to in vivo physiologic function.

Our study also has limitations. First, we focused on proximal, cartilaginous airways in order to test hypotheses about strands of mucus produced by submucosal glands. However, distal airways (beyond 10 generations in humans) lack submucosal glands but also contribute to MCT and will require different methods of investigation. Second, we studied the movement of large particles. Whether mucus strands also contribute to the movement of small particles in vivo remains uncertain. Third, DTT and TCEP will break disulfide bonds in MUC5AC and MUC5B produced by goblet cells as well as those in MUC5B arising from submucosal glands. However, our focus on mucus strands produced by submucosal glands limits that concern. An ideal experiment would be to eliminate MUC5B, but only in the submucosal glands of pigs; that approach is currently not feasible.

Defects in MCT have been reported in many airway diseases, including chronic obstructive pulmonary disease, primary ciliary dyskinesia, cystic fibrosis, and asthma (48-51). Moreover, mucolytic agents have been proposed as treatments (52-56). Our current studies do not address these issues. However, we note that the abundance of mucus, defective release of mucus from submucosal gland ducts, and/or altered properties of mucus might be beneficially altered by reducing agents. Moreover, specific defects in initiation or propagation of MCT might warrant specific therapeutic approaches. For instance, intermittent mucolytics might release obstructive plugs of mucus without eliminating the beneficial effects of mucus strands. The results of our study provide a foundation for further understanding how reducing agents affect MCT in heath and disease.

\section{Methods}

\section{Animals}

Newborn pigs were obtained from Exemplar Genetics. We studied male and female pigs 8-15 hours after birth. Sedation was with ketamine (20 mg/kg, i.m., Phoenix Pharmaceutical Inc.) and acepromazine ( $2 \mathrm{mg} / \mathrm{kg}$, i.m., Phoenix Pharmaceutical Inc.) or xylazine ( $2 \mathrm{mg} / \mathrm{kg}$, i.m., Lloyd), and anesthesia was maintained with i.v. dexmedetomidine (10 $\mu \mathrm{g} / \mathrm{kg} / \mathrm{h}$, i.v., Accord Healthcare Inc.). Euthanasia was with i.v. Euthasol (Virbac).

\section{Ex vivo methods}

Tissue preparation. 1-cm-long tracheal segments were removed immediately after euthanasia. A longitudinal cut was made along the ventral surface, and the tissue was pinned flat to expose the mucosal surface. The tissue was immediately flooded with $40 \mathrm{ml}$ Krebs-Ringer saline as previously described (20).

Experimental buffers and interventions. For most ex vivo studies, we used a $\mathrm{HCO}_{3}^{-} / \mathrm{CO}_{2}$ buffered Krebs-Ringer saline containing $118.9 \mathrm{mM} \mathrm{NaCl}, 25 \mathrm{mM} \mathrm{NaHCO}_{3}, 10 \mathrm{mM}$ dextrose, $2.4 \mathrm{mM} \mathrm{K}_{2} \mathrm{HPO}_{4}, 0.6$ $\mathrm{mM} \mathrm{KH}_{2} \mathrm{PO}_{4}, 1.2 \mathrm{mM} \mathrm{CaCl}_{2}, 1.2 \mathrm{mM} \mathrm{MgCl}_{2}, \mathrm{pH} 7.35$, with $5 \% \mathrm{CO}_{2}$ at $37^{\circ} \mathrm{C}$. In some preparations, 100 $\mu \mathrm{M}$ methacholine was added to stimulate submucosal gland secretion. In some experiments, $1 \mathrm{mM}$ DTT or $1 \mathrm{mM}$ TCEP was added to disrupt mucus strand formation. 
Imaging mucus with fluorescent nanospheres. We imaged secretion of mucus strands using 40-nm red or green fluorescent nanospheres (FluoSpheres, Molecular Probes) added to a final dilution of 1:10,000 as previously described (20). In some preparations, we used $\times 10$ (Nikon Plan Fluor, Dry, NA 0.3) and/or $\times 25$ (Nikon Apo LWD, Water, NA 1.1) objectives and a Nikon A1R confocal microscope. In these experiments, we acquired images at 8-10 frames per second for 15 minutes for each intervention. Observers blinded to experimental conditions counted the number of mucus strands that passed a predefined $140-\mu \mathrm{m}$ line placed orthogonal to the direction of ciliary flow. In some experiments, we added 4- $\mu \mathrm{m}$ fluorescent microspheres (Invitrogen) to indicate direction of flow.

Ciliary beat frequency. To measure ciliary beating, we used a $\times 25$ objective and acquired images at 110 frames per second. To determine ciliary beat frequency, we counted intensity oscillations over individual ciliated cells with time. We interrogated 5-6 cells per condition.

Ex vivo MCT assay. To assess MCT ex vivo, we added 0.6-mm steel spheres (SuperMagnetMan) or 0.5$\mathrm{mm}$ tantalum spheres (Bal-tec) onto the airway surface and acquired sequential images at 2.9-second intervals for 15 minutes (iPhone 5 Frameography application from Studio Neat). Quantitative data presented in this study represent experiments using Ta spheres. Individual spheres were tracked in Imaris software (Imaris v 7.6.4, Bitplane).

Analysis. We calculated the displacement of each sphere from its original position at each time point. Spheres that failed to move $>1 \mathrm{~mm}$ from their initial position and were observed for at least 10 minutes were labeled as non-starters. Using data pooled from 15 experiments, we compared the time required for spheres to travel $>1 \mathrm{~mm}$ under different experimental conditions using the log-rank test using the survival analysis feature of GraphPad Prism. We calculated the mean time spheres were $\leq 1 \mathrm{~mm}$ of their origin for each experiment and compared conditions by 2-way repeated-measures ANOVA. We reported the maximum and mean speed of spheres that moved greater than $1 \mathrm{~mm}$ during the duration of an experiment. We calculated the percentage of spheres that were cleared from the field as the number of spheres that reached the tissue edge divided by the total number of spheres added.

\section{In vivo methods}

In vivo MCT assay. To measure MCT in vivo, we used a previously described X-ray CT assay. We measured MCT by tracking tantalum microdisks ( $350-\mu \mathrm{m}$ diameter $\times 25-\mu \mathrm{m}$ thick, MilliporeSigma). To deliver microdisks, animals were anesthetized, briefly intubated, and microdisks were insufflated into the airways just beyond the vocal cords with a puff of air. Immediately after delivery, the tubes and catheter were removed. CT scans were acquired with a high-resolution multi-row detector computerized tomography scanner (Siemens Somatom, Force Dual Source 384-slice CT scanner). 44 CT scans were obtained in a 6.3-minute time interval. Microdisks were tracked over time by an automated algorithm (FiJi TrackMate plug-in, ref. 57) and manually validated. In some experiments, segments of trachea were removed immediately after euthanasia, opened on the ventral surface, flattened, and fixed in ice-cold 2\% paraformaldehyde overnight. Mucus was stained with 1:1000 rhodamine/WGA as previously reported (16) and imaged submerged on Nikon A1R confocal microscope.

CT scan analysis. Microdisks that failed to move $>10 \mathrm{~mm}$ from their initial position were labeled as non-starters. We recorded the time for each microdisk to travel $10 \mathrm{~mm}$ or the total time the microdisk was observed. Using data pooled from 7 experiments, we compared the time required for microdisks to travel $>10$ mm under different experimental conditions using survival analysis in GraphPad Prism. We calculated the mean time microdisks were $\leq 10 \mathrm{~mm}$ of their origin for each experiment and compared conditions by 1 -way ANOVA. Tracking microdisks over time provided multiple instantaneous measurements of microdisk speed. From these speeds, we determined individual microdisk maximum speeds and mean speeds for microdisks that moved $>10 \mathrm{~mm}$. Microdisk clearance was determined by measuring whether a microdisk left the tracking field or not during the 6.3-minute tracking period. The percentage of microdisks cleared was determined by dividing the number of cleared microdisks by the total number of microdisks tracked and multiplying by 100 .

\section{Statistics}

Data are presented as points from individual animals or tissue obtained from individual animals, with mean \pm SEM indicated by bars. We used D'Agostino-Pearson or Shapiro-Wilk tests to check for deviation from normality. For multiple comparisons, we used 1-way ANOVA or 2-way ANOVA with repeated measures. We used Holm-Sidak's Multiple Comparison test for post hoc correction. To compare time for spheres or microdisks to travel beyond a threshold, we used survival analysis using a log-rank test. In Figure 
$3 \mathrm{~F}$, we used a $\chi^{2}$ test. The Wilson/Brown method was used to calculate $95 \%$ CI. In Figure 4, E and F, we used Kruskal-Wallis test for nonnormally distributed data. Differences were considered statistically significant at $P<0.05$. All analyses were completed in GraphPad Prism v7.0d (GraphPad Software).

\section{Study approval}

The present studies in animals were reviewed and approved by the University of Iowa Animal Care and Use Committee.

\section{Author contributions}

AJF, MJW, and MHAA conceived and designed studies. AJF, MIPA, BMH, CRS, NDG, ALC, KZ, PDA, and MHAA conducted experiments and acquired data. AJF, MIPA, LSO, EAH, DAS, MJW, and MHAA analyzed data. AJF, MJW, and MHAA wrote the manuscript.

\section{Acknowledgments}

We thank Theresa M. Chapdelaine and Mingxia Xu for writing code to analyze MCT. We thank Lokhoy Brecht for help with the manuscript preparation. This work was funded in part by NIH grant K08 HL136927 and Cystic Fibrosis Foundation (CFF) grant FISCHE16I0 (to AJF), NIH grant K08 HL135433 (to MHAA), CFF grant STOLTZ16XX0 (to DAS and MHAA), NIH grant R01 HL136813 (to DAS), and NIH Program Project Grant HL091842 and HL051670 (to DAS and MJW). MJW is an investigator of the Howard Hughes Medical Institute.

Address correspondence to: Anthony J. Fischer, Carver College of Medicine, Department of Pediatrics, 6312 PBDB, University of Iowa, Iowa City, Iowa 52242, USA. Phone: 319.335.6992; Email: anthony-fischer@uiowa.edu. Or to: Mahmoud H. Abou Alaiwa, Carver College of Medicine, Department of Internal Medicine, 6338 PBDB, University of Iowa, Iowa City, Iowa 52242, USA. Phone: 319.384.1107; Email: mahmoud-aboualaiwa@uiowa.edu.

1. Wanner A, Salathé M, O'Riordan TG. Mucociliary clearance in the airways. Am J Respir Crit Care Med. 1996;154(6 Pt 1):1868-1902.

2. Houtmeyers E, Gosselink R, Gayan-Ramirez G, Decramer M. Regulation of mucociliary clearance in health and disease. Eur Respir J. 1999;13(5):1177-1188.

3. Fahy JV, Dickey BF. Airway mucus function and dysfunction. N Engl J Med. 2010;363(23):2233-2247.

4. Thornton DJ, Rousseau K, McGuckin MA. Structure and function of the polymeric mucins in airways mucus. Annu Rev Physiol. 2008;70:459-486.

5. Hovenberg HW, Davies JR, Carlstedt I. Different mucins are produced by the surface epithelium and the submucosa in human trachea: identification of MUC5AC as a major mucin from the goblet cells. Biochem J. 1996;318(Pt 1):319-324

6. Widdicombe JH, Wine JJ. Airway gland structure and function. Physiol Rev. 2015;95(4):1241-1319.

7. Rose MC, Voynow JA. Respiratory tract mucin genes and mucin glycoproteins in health and disease. Physiol Rev. 2006;86(1):245-278.

8. Thornton DJ, Davies JR, Kraayenbrink M, Richardson PS, Sheehan JK, Carlstedt I. Mucus glycoproteins from 'normal' human tracheobronchial secretion. Biochem J. 1990;265(1):179-186.

9. Hovenberg HW, Davies JR, Herrmann A, Lindén CJ, Carlstedt I. MUC5AC, but not MUC2, is a prominent mucin in respiratory secretions. Glycoconj J. 1996;13(5):839-847.

10. Wickström C, Davies JR, Eriksen GV, Veerman EC, Carlstedt I. MUC5B is a major gel-forming, oligomeric mucin from human salivary gland, respiratory tract and endocervix: identification of glycoforms and C-terminal cleavage. Biochem J. 1998;334(Pt 3):685-693

11. Okuda K, et al. Localization of secretory mucins MUC5AC MUC5B in normal/healthy human airways [published online ahead of print October 23, 2018]. Am J Respir Crit Care Med. https://doi.org/10.1164/rccm.201804-0734OC.

12. Choi HK, Finkbeiner WE, Widdicombe JH. A comparative study of mammalian tracheal mucous glands. $J$ Anat. $2000 ; 197 \mathrm{Pt}$ 3:361-372.

13. Whimster WF. Distribution of submucous gland in the human trachea, main and segmental bronchi: morphometric study. Appl Pathol. 1986;4(1-2):15-23.

14. Whimster WF. Number and mean volume of individual submucous glands in the human tracheobronchial tree. Appl Pathol. 1986;4(1-2):24-32.

15. Plopper CG, Heidsiek JG, Weir AJ, George JA, Hyde DM. Tracheobronchial epithelium in the adult rhesus monkey: a quantitative histochemical and ultrastructural study. Am J Anat. 1989;184(1):31-40.

16. Ostedgaard LS, et al. Gel-forming mucins form distinct morphologic structures in airways. Proc Natl Acad Sci USA 2017;114(26):6842-6847.

17. Phipps RJ, Richardson PS. The effects of irritation at various levels of the airway upon tracheal mucus secretion in the cat. $J$ Physiol (Lond). 1976;261(3):563-581. 
18. Wine JJ. Parasympathetic control of airway submucosal glands: central reflexes and the airway intrinsic nervous system. Auton Neurosci. 2007;133(1):35-54

19. Choi JY, et al. Substance P stimulates human airway submucosal gland secretion mainly via a CFTR-dependent process. JClin Invest. 2009;119(5):1189-1200.

20. Hoegger MJ, et al. Impaired mucus detachment disrupts mucociliary transport in a piglet model of cystic fibrosis. Science. 2014;345(6198):818-822.

21. Ermund A, et al. The normal trachea is cleaned by MUC5B mucin bundles from the submucosal glands coated with the MUC5AC mucin. Biochem Biophys Res Commun. 2017;492(3):331-337.

22. Trillo-Muyo S, et al. Granule-stored MUC5B mucins are packed by the non-covalent formation of N-terminal head-to-head tetramers. J Biol Chem. 2018;293(15):5746-5754.

23. Oppenheimer EH. Similarity of the tracheobronchial mucous glands and epithelium in infants with and without cystic fibrosis. Hum Pathol. 1981;12(1):36-48.

24. Simel DL, et al. Scanning electron microscopic study of the airways in normal children and in patients with cystic fibrosis and other lung diseases. Pediatr Pathol. 1984;2(1):47-64.

25. Thornton DJ, et al. Salivary mucin MG1 is comprised almost entirely of different glycosylated forms of the MUC5B gene product. Glycobiology. 1999;9(3):293-302.

26. Cline DJ, Redding SE, Brohawn SG, Psathas JN, Schneider JP, Thorpe C. New water-soluble phosphines as reductants of peptide and protein disulfide bonds: reactivity and membrane permeability. Biochemistry. 2004;43(48):15195-15203.

27. Rogers CS, et al. The porcine lung as a potential model for cystic fibrosis. Am J Physiol Lung Cell Mol Physiol. 2008;295(2):L240-L263.

28. Eliezer N, Sadé J, Silberberg A, Nevo AC. The role of mucus in transport by cilia. Am Rev Respir Dis. 1970;102(1):48-52.

29. Hoegger MJ, et al. Assessing mucociliary transport of single particles in vivo shows variable speed and preference for the ventral trachea in newborn pigs. Proc Natl Acad Sci USA. 2014;111(6):2355-2360.

30. Nadel JA, Davis B, Phipps RJ. Control of mucus secretion and ion transport in airways. Annu Rev Physiol. 1979;41:369-381.

31. Widdicombe JH, Chen LL, Sporer H, Choi HK, Pecson IS, Bastacky SJ. Distribution of tracheal and laryngeal mucous glands in some rodents and the rabbit. J Anat. 2001;198(Pt 2):207-221.

32. Roy MG, et al. Muc5b is required for airway defence. Nature. 2014;505(7483):412-416

33. Grubb BR, Livraghi-Butrico A, Rogers TD, Yin W, Button B, Ostrowski LE. Reduced mucociliary clearance in old mice is associated with a decrease in Muc5b mucin. Am J Physiol Lung Cell Mol Physiol. 2016;310(9):L860-L867.

34. Zhu Y, et al. Munc13-2-/- baseline secretion defect reveals source of oligomeric mucins in mouse airways. J Physiol (Lond). 2008;586(7):1977-1992.

35. Bermbach S, et al. Mechanisms of cilia-driven transport in the airways in the absence of mucus. Am J Respir Cell Mol Biol. 2014;51(1):56-67.

36. Hirsch SR, Zastrow JE, Kory RC. Sputum liquefying agents: a comparative in vitro evaluation. J Lab Clin Med. 1969;74(2):346-353.

37. Martin R, Litt M, Marriott C. The effect of mucolytic agents on the rheologic and transport properties of canine tracheal mucus. Am Rev Respir Dis. 1980;121(3):495-500.

38. Tomkiewicz RP, App EM, Coffiner M, Fossion J, Maes P, King M. Mucolytic treatment with N-acetylcysteine L-lysinate metered dose inhaler in dogs: airway epithelial function changes. Eur Respir J. 1994;7(1):81-87.

39. Seagrave J, Albrecht HH, Hill DB, Rogers DF, Solomon G. Effects of guaifenesin, N-acetylcysteine, and ambroxol on MUC5AC and mucociliary transport in primary differentiated human tracheal-bronchial cells. Respir Res. 2012;13:98.

40. Tang XX, et al. Acidic pH increases airway surface liquid viscosity in cystic fibrosis. J Clin Invest. 2016;126(3):879-891.

41. Puchelle E, Girard F, Zahm JM. [Rheology of bronchial secretions and mucociliary transport (author's transl)]. Bull Eur Physiopathol Respir. 1976;12(6):771-779.

42. Giordano A, Holsclaw D, Litt M. Effects of various drugs on canine tracheal mucociliary transport. Ann Otol Rhinol Laryngol. 1978;87(4 Pt 1):484-490

43. Melville GN, Ismail S, Sealy C. Tracheobronchial function in health and disease. Effect of mucolytic substances. Respiration. 1980;40(6):329-336

44. Todisco T, et al. Effect of N-acetylcysteine in subjects with slow pulmonary mucociliary clearance. Eur J Respir Dis Suppl. 1985;139:136-141.

45. Olivieri D, Marsico SA, Del Donno M. Improvement of mucociliary transport in smokers by mucolytics. Eur J Respir Dis Suppl. 1985;139:142-145.

46. Hosoe H, et al. Mucolytic and antitussive effects of erdosteine. J Pharm Pharmacol. 1999;51(8):959-966.

47. Iravani J, Melville GN, Horstmann G. N-Acetylcysteine and mucociliary activity in mammalian airways. Arzneimittelforschung. 1978;28(2):250-254.

48. Aikawa T, Shimura S, Sasaki H, Takishima T, Yaegashi H, Takahashi T. Morphometric analysis of intraluminal mucus in airways in chronic obstructive pulmonary disease. Am Rev Respir Dis. 1989;140(2):477-482

49. Bush A, Payne D, Pike S, Jenkins G, Henke MO, Rubin BK. Mucus properties in children with primary ciliary dyskinesia: comparison with cystic fibrosis. Chest. 2006;129(1):118-123.

50. Raju SV, et al. The cystic fibrosis transmembrane conductance regulator potentiator ivacaftor augments mucociliary clearance abrogating cystic fibrosis transmembrane conductance regulator inhibition by cigarette smoke. Am J Respir Cell Mol Biol. 2017;56(1):99-108.

51. Dunican EM, et al. Mucus plugs in patients with asthma linked to eosinophilia and airflow obstruction. J Clin Invest. 2018;128(3):997-1009.

52. Tam J, Nash EF, Ratjen F, Tullis E, Stephenson A. Nebulized and oral thiol derivatives for pulmonary disease in cystic fibrosis. Cochrane Database Syst Rev. 2013;(7):CD007168.

53. Nash EF, Stephenson A, Ratjen F, Tullis E. Nebulized and oral thiol derivatives for pulmonary disease in cystic fibrosis. Cochrane Database Syst Rev. 2009;(1):CD007168

54. Duijvestijn YC, Brand PL. Systematic review of N-acetylcysteine in cystic fibrosis. Acta Paediatr. 1999;88(1):38-41.

55. Ayfer Aytemur Z, Baysak A, Ozdemir O, Köse T, Sayiner A. N-acetylcysteine in patients with COPD exacerbations associated 
with increased sputum. Wien Klin Wochenschr. 2015;127(7-8):256-261.

56. Aliyali M, Poorhasan Amiri A, Sharifpoor A, Zalli F. Effects of N-acetylcysteine on asthma exacerbation. Iran J Allergy Asthma Immunol. 2010;9(2):103-109.

57. Tinevez JY, et al. TrackMate: An open and extensible platform for single-particle tracking. Methods. 2017;115:80-90. 